\title{
Preparation of heterocyclic compounds by reaction of dimethyl and diethyl acetylene dicarboxylate (DMAD, DEAD) with thiosemicarbazone derivatives
}

\author{
Ali Darehkordi ${ }^{a}$, Kazem Saidi ${ }^{\mathbf{b}^{*}}$, and Mohammad Reza Islami ${ }^{\mathrm{b}}$ \\ ${ }^{a}$ Department of Chemistry, Vali-e-asr University of Rafsanjan, Rafsanjan, Iran \\ ${ }^{b}$ Department of Chemistry, Shahid Bahonar University of Kerman, Kerman 76169, Iran \\ E-mail: saidik@mail.uk.ac.ir
}

\begin{abstract}
Different thiosemicarbazone derivatives react with dimethyl acetylene dicarboxylate (DMAD) and diethyl acetylene dicarboxylate (DEAD) by three different methods: a) in ethyl acetate solvent at ambient temperature, b) one-pot synthesis under microwave irradiation and solventfree conditions (three-component reaction between thiosemicarbazide, aldehyde/ ketone and DMAD or DEAD), c) a microwave-assisted synthesis under solvent-free conditions, to obtain five-membered $\mathrm{S}, \mathrm{N}$-heterocycles thiazolines in good to excellent yields.
\end{abstract}

Keywords: Thiosemicarbazone, dimethyl acetylene dicarboxylate, diethyl acetylene dicarboxylate, thiazoline

\section{Introduction}

Reaction of dimethyl acetylene dicarboxylate (DMAD) with esters and amides of dithiocarboxylic acids are well known methods for preparation of five membered S, and S,Nheterocycles ${ }^{1,2}$. Thioureas possessing more than two N-H bonds react with (DMAD) to give 1:1 molar-methanol adducts ${ }^{2}$. McKillop et al. have reported that benzimidazole-2 -thione reacted with DMAD in either methanol or acetic acid to give mixture of two 1:1 molar-methanol one adduct which was identified as [Type (1)] by X-ray crystallography but other adduct which was not isolated was only tentatively assigned structure (2) from its ${ }^{1} \mathrm{H}$ NMR spectrum ${ }^{2,5}$. The application of microwave irradiation in organic synthesis for conducting reactions at highly accelerated rates is an emerging technique ${ }^{3}$. In fact, in recent years, the use of microwave has become popular among synthetic organic chemists both to improve classical organic reactions (shortening reaction times and /or improving yield) as well as to promote new reactions ${ }^{6}$. 


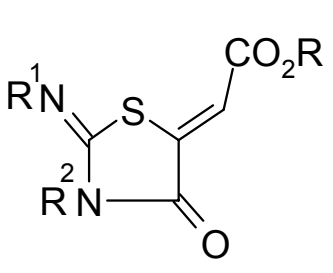

(1)

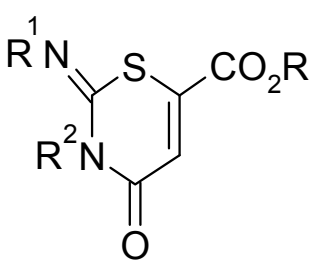

(2)

We have considered the reaction of thiosemicarbazone derivatives with dimethyl acetylene dicarboxylate and diethyl acetylene dicarboxylate to synthesis thiazolines, and to find the influence of the nature of electron donor group on the progress of this reaction, in addition on the structure of the resulting products. It should be noted that the reaction of thiosemicarbazone derivatives which contain two $\mathrm{N}-\mathrm{H}, \mathrm{NH}_{2}$ and one S-group with DMAD and DEAD have not been described in the literature thus far.

\section{Results and Discussion}

In continuing our interest in the synthesis of heterocyclic compounds such as 1,3-thiazinones ${ }^{7}$ and other heterocyclic compounds. We would like now to report the synthesis of thiazoline compounds from thiosemicarbazone derivatives possessing two $\mathrm{N}-\mathrm{H}, \mathrm{NH}_{2}$ groups and one $\mathrm{S}=\mathrm{C}$ bond which react with DMAD and DEAD to give thiazoline. In this reaction the sulfur atom attacked the triple bonds and a thiolactam is formed, followed by the aminolyses of an ester group $^{8}$. This reaction can lead to the structures type (1), and type (2). Both products have been claimed, although structure type 2 has not been confirmed. In this investigation we report three methods for the synthesis of thiazolines: a) thiosemicarbazone is dissolved in ethyl acetate then DMAD or DEAD is added to this solution, reaction was carried out with a stirring for $2.5-5 \mathrm{hr}$ at ambient temperature, b) a facile synthesis via a one-pot three-component reaction between thiosemicarbazide, aldehyde/ketone and DMAD or DEAD in the present of catalytic amounts of acetic acid, thiosemicarbazide and aldehydes are converted in-situ to thiosemicarbazone, next the thiosemicarbazone are reacted with DMAD or DEAD under microwave irradiation and solventfree condition to produce thiazolin in $95-98 \%$ yields, c) in the third method, thiosemicarbazone was mixed with DMAD or DEAD, then the reaction was subject to microwave irradiation under free solvent conditions at short experimental time. The following results were obtained, formation of $1 \mathrm{c}$, after $3 \mathrm{~h}$ in method a, $3 \mathrm{~min}$ in method $\mathrm{b}$ and $5 \mathrm{~min}$ in method c. A plausible mechanism is shown below, scheme (1). Compound 1c reveals $\mathrm{NH}$ group at $3180, \mathrm{C}-\mathrm{H}$ absorption of thiophene ring at 2780-3081 $\mathrm{cm}^{-1}$, carbonyl groups at $1741,1661 \mathrm{~cm}^{-1}$ and $\mathrm{C}=\mathrm{N}$, $\mathrm{C}=\mathrm{C}$ at $1666,1641,1617 \mathrm{~cm}^{-1}$ in the IR spectrum. The ${ }^{1} \mathrm{H}$ NMR spectrum of $1 \mathrm{c}$ indicated one singlet quite down field at $(\delta 12.60 \mathrm{ppm})$ which is the proton of $\mathrm{NH}$ amide, thiophene ring protons at 7.75, 7.57, 7.18, vinyl proton at 6.64 and methoxy protons at $3.76 \mathrm{ppm}$. The ${ }^{13} \mathrm{C}$ NMR spectrum of $1 \mathrm{c}$ indicated carbonyl group carbons at $165.69,165.48,159.16(\mathrm{C}=\mathrm{N}), 152.77(\mathrm{~S}-$ 
$\mathrm{C}=\mathrm{CH}), 142.58(\mathrm{C}=\mathrm{CH}$ of thiophene ring $), 138.06(\mathrm{CH}=\mathrm{N}), 132.99,130.59,128.03(\mathrm{C}=\mathrm{CH}$ of thiophene ring), $113.48(\mathrm{C}=\mathrm{CH})$ and methoxy carbon at $52.23 \mathrm{ppm}$. Mass spectral data and elemental analyses are also in accordance with the proposed structure.

In general all of the spectral data support the structures for the compounds (1c-10c), Table 1.

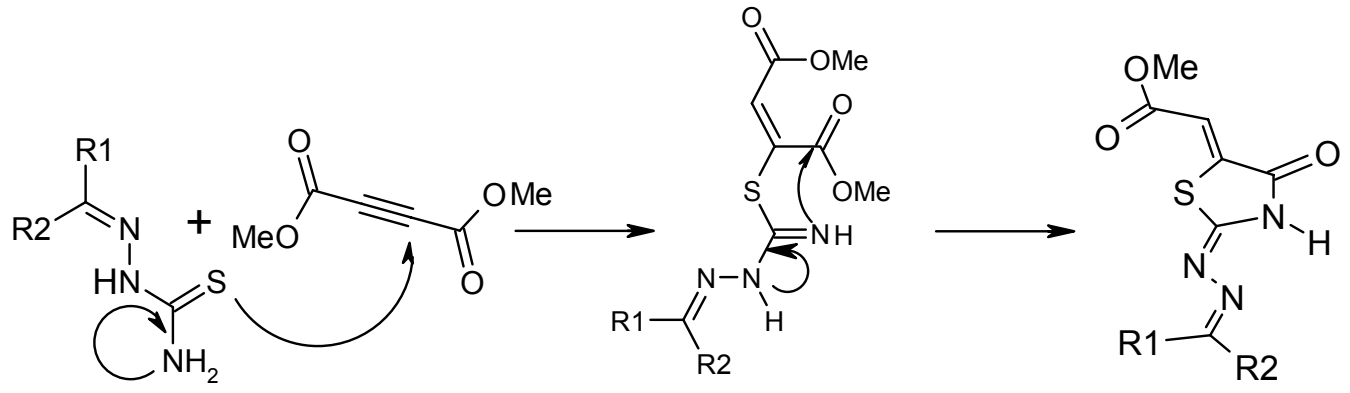

a

b

C

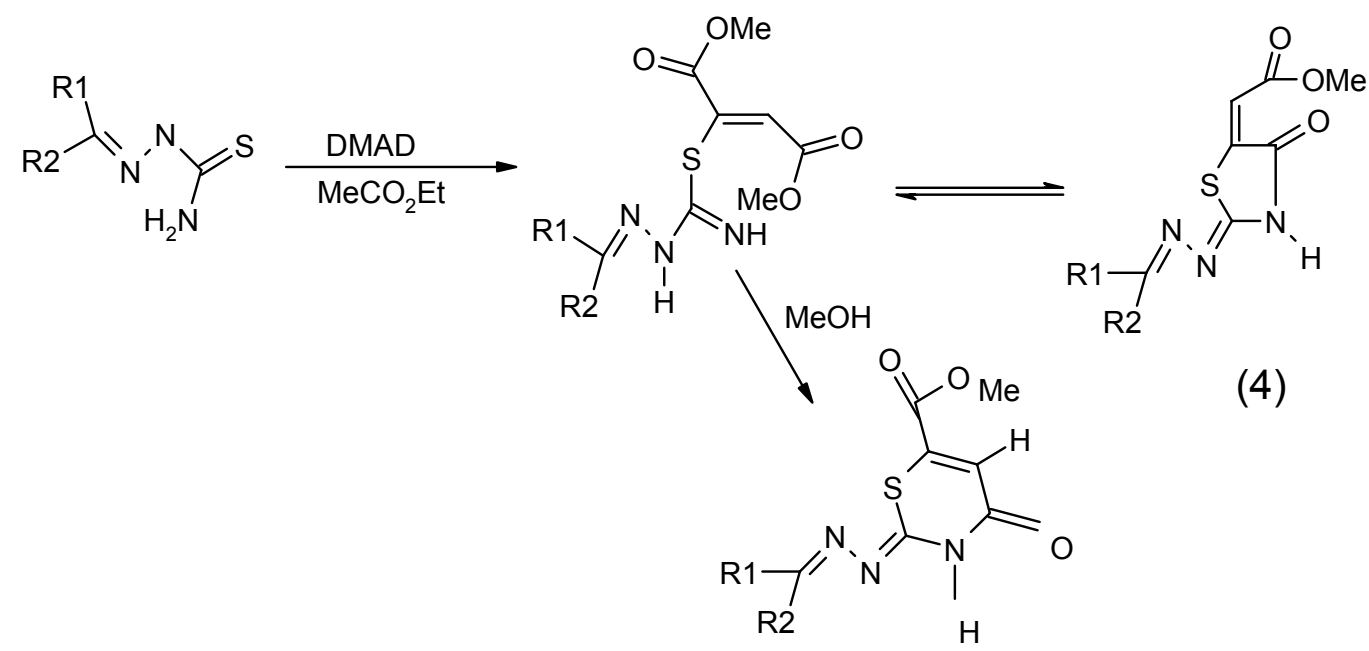

(5)

\section{Scheme 1}

We have found that the thiosemicarbazone, DMAD and DEAD reacted in ethyl acetate to give only structure (4), and when they were reacted in dry methanol only structure (5) was formed. The adduct (4) was converted to (5) by refluxing the reaction mixture in dry methanol. This rearrangement was probably catalyzed by a base. Since, it did not take place if the methanol contained a few drops of acetic acid. A possible mechanism (Scheme 2) involves ring-opening by attack of methoxide on the strained cyclic amide, followed by ring-closure on the other ester group. 


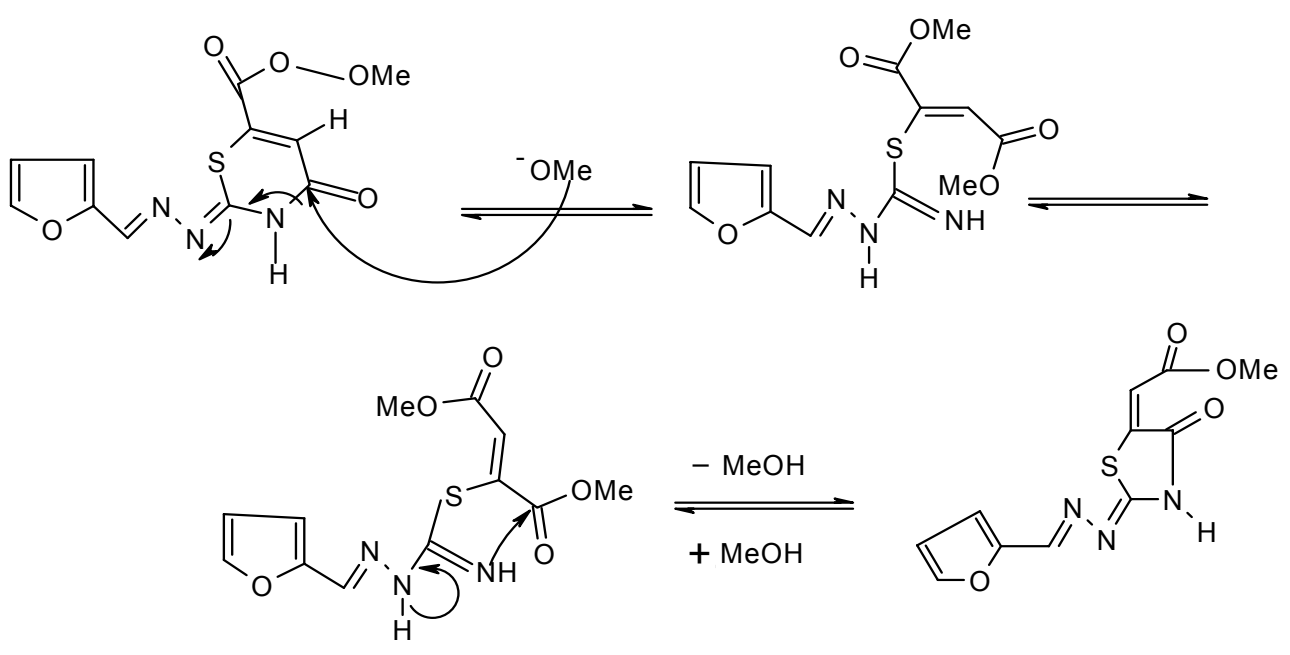

The effect of the electron-donor group on the progress of the reaction and on the structure of the final products was also investigated. When such an electron donor group is present on the benzene ring higher yields were obtained without exerting heat or reflux the reaction mixture, and in addition the reaction rate is much faster.<smiles>COC(=O)/C=C1\S/C(=N/N=C/c2ccc(OC)cc2)NC1=O</smiles><smiles>COC(=O)/C=C1\S/C(=N/N=C/c2ccc(OC)cc2)NC1=O</smiles>

\section{Scheme 2}


Table 1. Compounds and yields

\begin{tabular}{|c|c|c|c|c|}
\hline Compound & Structure of compound & $\begin{array}{l}\text { Isolated } \\
\text { yield }(\%) \\
\text { method a }\end{array}$ & $\begin{array}{l}\text { Isolated yield }(\%) \\
\text { method } b\end{array}$ & $\begin{array}{l}\text { Isolated } \\
\text { yield (\%) } \\
\text { method c }\end{array}$ \\
\hline 1c & & 78 & 85 & 82 \\
\hline $2 c$ & & 80 & 83 & 82 \\
\hline $3 \mathbf{c}$ & & 80 & 89 & 90 \\
\hline $4 c$ & & 93 & 92 & 95 \\
\hline $5 c$ & & 87 & 91 & 94 \\
\hline $6 c$ & & 86 & 87 & 90 \\
\hline $7 \mathrm{c}$ & & 87 & 88 & 90 \\
\hline $8 c$ & & 90 & 92 & 94 \\
\hline 9c & & 85 & 87 & 89 \\
\hline
\end{tabular}




\section{Conclusions}

Since the use of microwave are playing increasingly pivotal role to synthesis organic compounds, we have developed a microwave-assisted, one-pot, three-component reaction for the preparation of thiazolines with excellent yield. No harsh conditions or cumbersome apparatus are required. Simple purification process, short experimental time for the reaction to be completed and solvent free conditions are the main advantages of these methods, in addition these reactions require the environmentally friendly conditions.

\section{Experimental Section}

Furfural, thiofurfural, benzophenone, butanal, $p$-methoxybenzaldehyde, $p$-methylacetophenone, $p$-methyl benzaldehyde, 2-ketoethylbutyrate, $p$ - $N, N$-dimethybenzaldehyde, thiosemicarbazide, sodium acetate , dimethyl acetylene dicarboxylate (DMAD), and diethyl acetylene dicarboxylate (DEAD) and all the solvents such as ethyl acetate and ethanol were purchased from Merck chemical co. and were used without further purification. Thiosemicarbazones were prepared according to the procedure in the literature ${ }^{7}$. IR spectra were obtained on a Matson-1000 FT-IR spectrometer. The proton and carbon-13 NMR spectra were recorded with a BRUKER DRX-500 AVANCE spectrometer at 500 and $125.77 \mathrm{MHz}$ respectively with $\mathrm{Me}_{4} \mathrm{Si}$ as an internal standard. Elemental analyses were performed by National Iranian Oil Company lab (Tehran) using a Heracus CHN-O-Rapid analyzer. Mass spectrometer operating at an ionization potential of 70 $\mathrm{eV}$.

\section{General experimental procedures}

(a) To a solution of thiosemicarbazone-2-keto ethyl butyrate $(0.76 \mathrm{~g}, 4 \mathrm{mmol})$ in ethyl acetate $(100 \mathrm{ml})$ was added a solution of dimethyl acetylene dicarboxylate $(0.49 \mathrm{ml}, 4 \mathrm{mmol})$ in small portions. The solution was stirred at ambient temperature for $3 \mathrm{~h}$. The resulting yellow precipitate was filtered, washed with ethyl acetate, a yellow solid was separated which was then recrystallized from ethanol-water. yield $(1.04 \mathrm{~g}) 95 \%$.

(b) A mixture of thiosemicarbazide $(0.73 \mathrm{~g}, 8 \mathrm{mmol})$, p-methoxybenzaldehyde $(1.09 \mathrm{~g}, 8 \mathrm{mmol})$ and DMAD $(0.98 \mathrm{ml}, 8 \mathrm{mmol})$ and a catalytic amount of acetic acid was subject to microwave irradiation under microwave at $80 \mathrm{~W}$ for $3 \mathrm{~min}$. After cooling the reaction mixture to ambient temperature the solid residue was recrystallized from ethanol-water. The product was obtained as yellow crystals.

c)A mixture of thiosemicarbazone p-methoxybenzaldehyde ( $0.72 \mathrm{~g}, 4 \mathrm{mmol})$ and DMAD (0.49 $\mathrm{ml}, 4 \mathrm{mmol}$ ), was subject to microwave irradiation at $50 \mathrm{~W}$ for $5 \mathrm{~min}$, after cooling the reaction mixture to room temperature, the solid residue was recrystallized from ethanol-water. The product was obtained as yellow crystals. 
[4-Oxo-2-(thiophen-2-ylmethylene-hydrazono)thiazolidine]-acetic acid methyl ester (1c). Yellow solid, $0.92 \mathrm{~g}$, yield 78\%, m.p $>220{ }^{0} \mathrm{C}$ IR $(\mathrm{KBr})\left(\ddot{\mathrm{v}}_{\max }, \mathrm{cm}^{-1}\right): 2780-3081(\mathrm{CH}$, thiophene ring), 1741, $1716(2 \mathrm{C}=\mathrm{O}), 1661,1641,1617(\mathrm{C}=\mathrm{N}, \mathrm{C}=\mathrm{C}),{ }^{1} \mathrm{H}$ NMR $(\mathrm{DMSO}) \delta: 12.60(1 \mathrm{H}, \mathrm{s}$, $\mathrm{NH}), 8.60(1 \mathrm{H}, \mathrm{s}, \mathrm{CH}=\mathrm{N}), 7.75(1 \mathrm{H}, \mathrm{d}), 7.57(1 \mathrm{H}, \mathrm{d}), 7.18(1 \mathrm{H}, \mathrm{t})$, thiophene ring, $6.64(1 \mathrm{H}, \mathrm{s}$, $\mathrm{CH}=), 3.75(3 \mathrm{H}, \mathrm{s},-\mathrm{OMe}) .{ }^{13} \mathrm{C}$ NMR $(\mathrm{DMSO}) \delta$ : 165.69, $165.48(2 \mathrm{C}=\mathrm{O}), 159.16(\mathrm{C}=\mathrm{N}), 152.77$ $(\mathrm{S}-\mathrm{C}=\mathrm{CH}), 142.58$ (thiophene),138.06 $(\mathrm{CH}=\mathrm{N}), 132.99,130.59,128.03(\mathrm{C}=\mathrm{CH}$ of thiophene ring), $113.48(\mathrm{C}=\mathrm{CH}), 52.23\left(-\mathrm{OCH}_{3}\right) . \mathrm{MS}(\mathrm{m} / \mathrm{z}, \%)$ : 295 (parent peak), 96 (base peak, 100\%). Anal. Calcd for $\mathrm{C}_{11} \mathrm{H}_{9} \mathrm{~N}_{3} \mathrm{O}_{3} \mathrm{~S}_{2}$ : C, 44.74; H, 3.05; N, 14.24\%. Found; C, 44.60; H, 2.95; N, $14.13 \%$.

[2-(Furan-2-ylmethylene-hydrazono)-4-oxo-thiazolidin-5-ylidene]-acetic acid methyl ester (2c). Yellow solid, $0.84 \mathrm{~g}$; yield $80 \%$, m.p $>220{ }^{0} \mathrm{C}$.IR $(\mathrm{KBr})\left(\hat{v}_{\max }, \mathrm{cm}^{-1}\right): 2800-3090(\mathrm{CH}$, furan ring), 1742, $1717(\mathrm{C}=\mathrm{O}), 1667,1645,1617(\mathrm{C}=\mathrm{N}, \mathrm{C}=\mathrm{C}),{ }^{1} \mathrm{H}$ NMR (DMSO) $\delta: 12.75$ (1H, s, $\mathrm{NH}), 8.33(1 \mathrm{H}, \mathrm{s}, \mathrm{CH}=\mathrm{N}), 7.90,7.05,6.67(3 \mathrm{H}$, furan ring), $6.63(1 \mathrm{H}, \mathrm{s}, \mathrm{CH}=\mathrm{C}), 3.77(3 \mathrm{H}, \mathrm{s},-$ OMe). ${ }^{13} \mathrm{C}$ NMR (DMSO) $\delta: 165.85,165.00(2 \mathrm{C}=\mathrm{O}), 160.00,148.88(\mathrm{C}=\mathrm{N}), 147.53(\mathrm{C}=\mathrm{CH})$, 146.55, 142.77, $117.17,114.5$ (furan-ring), $112.56(\mathrm{C}=\mathrm{CH}), 52.43(1 \mathrm{C},-\mathrm{OMe})$. Anal. Calcd for $\mathrm{C}_{11} \mathrm{H}_{9} \mathrm{~N}_{3} \mathrm{O}_{4} \mathrm{~S}$ : C, 46.64; H, 3.18; N, 14.84\%. Found; C, 46.39; H, 3.15; N, 14.49\%.

\{2-[(4-Dimethylamino-benzylidene)-hydrazono]-4-oxo-thiazolidin-5-ylidene\} acetic acid methyl ester (3c). Orange solid, $1.06 \mathrm{~g}$, yield $80 \%$, m.p $>220{ }^{0} \mathrm{C}$. IR (KBr) $\left(\ddot{v}_{\max }, \mathrm{cm}^{-1}\right): 3020$ (C-H, arom), 1735, $1715(\mathrm{C}=\mathrm{O}), 1643,1615(\mathrm{C}=\mathrm{N}),{ }^{1} \mathrm{H}$ NMR (DMSO) $\delta: 8.23(1 \mathrm{H}, \mathrm{s}, \mathrm{CH}=\mathrm{N})$, 7.5-6.73 (4H, para, aromatic), $6.40(1 \mathrm{H}, \mathrm{CH}=), 3.72(3 \mathrm{H}, \mathrm{s},-\mathrm{OMe}), 2.96\left(6 \mathrm{H}, \mathrm{s}, 2-\mathrm{CH}_{3}\right) .{ }^{13} \mathrm{C}$ NMR (DMSO) $\delta: 172.06,166.81(2 \mathrm{C}=\mathrm{O}), 154.77(\mathrm{C}=\mathrm{N}), 151.67-111.79(\mathrm{C}=\mathrm{C}), 51.80(-\mathrm{OMe})$, $21.07\left(-\mathrm{CH}_{3}\right)$. MS (m/z, \%): 332 (parent peak), 332 (base peak, 100\%), 147, 133, 118, 44. Anal. Calcd for $\mathrm{C}_{15} \mathrm{H}_{16} \mathrm{~N}_{4} \mathrm{O}_{3} \mathrm{~S}$ : C, 54.22; H, 4.82; N, 16.87\%. Found; C, 54.16; H, 4.57; N, 16.67\%.

\{2-[(4-Methoxy-benzylidene)-hydrazono]-4-oxo-thiazolidin-5-ylidene\}-acetic acid methyl ester (4c).Yellow solid, $1.19 \mathrm{~g}$, yield 93\%, m.p $>220{ }^{0} \mathrm{C}$. IR $(\mathrm{KBr})\left(\hat{\mathrm{v}}_{\max }, \mathrm{cm}^{-1}\right)$ 2982-3081 (C-H, arom)1741, $1716(\mathrm{C}=\mathrm{O}), 1666,1617(\mathrm{C}=\mathrm{N}),{ }^{1} \mathrm{H}$ NMR (DMSO) $\delta: 12.40(1 \mathrm{H}, \mathrm{s}, \mathrm{NH}), 9.67(1 \mathrm{H}$, $\mathrm{s}, \mathrm{CH}=\mathrm{N}), 8.23(1 \mathrm{H}, \mathrm{s}, \mathrm{CH}=\mathrm{N}), 7.07-7.61(4 \mathrm{H}, \mathrm{m}$, arom), $6.70(1 \mathrm{H}, \mathrm{s}, \mathrm{CH}=\mathrm{C}), 3.90,3.63(6 \mathrm{H}, 2-$ OMe). ${ }^{13} \mathrm{C}$ NMR (DMSO) $\delta: 172.53,166.81,(2 \mathrm{C}=\mathrm{O}), 161.20,160.32(\mathrm{CH}=\mathrm{N}), 160.26-117.35$ (6C, m, arom), 148.32, $115.02(\mathrm{C}=\mathrm{CH}), 55.12,51.61(2-\mathrm{OMe}) . \mathrm{MS}(\mathrm{m} / \mathrm{z}, \%): 319$ (parent peak), 319 (base peak, 100\%), 134, 120, 77. Anal. Calcd for $\mathrm{C}_{14} \mathrm{H}_{13} \mathrm{~N}_{3} \mathrm{O}_{4} \mathrm{~S}: \mathrm{C}, 52.66 ; \mathrm{H}, 4.07 ; \mathrm{N}$, $13.17 \%$. Found; C, 52.80; H, 4.01; N, 13.01\%.

[2-(Benzhydrylidene-hydrazono)-4-oxo-thiazolidin-5-ylidene]-acetic acid mehyl ester (5c).Yellow solid, $1.27 \mathrm{~g}$, yield 87\%, m.p138-140, IR (KBr) $\left(\ddot{v}_{\max }, \mathrm{cm}^{-1}\right)$ : 2982-3000 (C-H, arom), 1741, $1716(\mathrm{C}=\mathrm{O}), 1642,1617(\mathrm{C}=\mathrm{N}), \mathrm{H}$ NMR (DMSO) $\delta: 8.60(1 \mathrm{H}, \mathrm{s}, \mathrm{NH}), 7.68-7.25$ $\left(10 \mathrm{H}, \mathrm{m}\right.$, arom), $6.80(1 \mathrm{H}, \mathrm{s}, \mathrm{CH}=\mathrm{C}), 3.86(3 \mathrm{H}, \mathrm{s},-\mathrm{OMe}) .{ }^{13} \mathrm{C}$ NMR (DMSO) $\delta: 166.42,165.26$ $(2 \mathrm{C}=\mathrm{O}), 165.21,160.74(2 \mathrm{C}=\mathrm{N}), 150.98-116.06(\mathrm{C}=\mathrm{CH}), 52.52(-\mathrm{OMe}) . \mathrm{MS}(\mathrm{m} / \mathrm{z}, \%): 365$ (parent peak), 165 (base peak, 100\%), 180, 77. Anal. Calcd for $\mathrm{C}_{19} \mathrm{H}_{15} \mathrm{~N}_{3} \mathrm{O}_{3} \mathrm{~S}: \mathrm{C}, 62.46 ; \mathrm{H}, 4.11$; N, 11.51\%. Found; C, 62.34; H, 11.35; N, 4.11\%.

[2-(Butylidene-hydrazono)-4-oxo-thiazolidin-5-ylidene]-acetic acid methyl ester (6c).Yellow

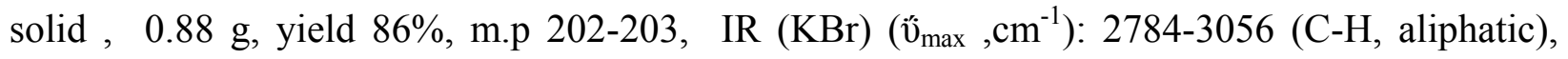


1741, $1666(\mathrm{C}=\mathrm{O}), 1641,1617(\mathrm{C}=\mathrm{N}),{ }^{1} \mathrm{H}$ NMR (DMSO) $\delta: 12.43(1 \mathrm{H}, \mathrm{s}, \mathrm{NH}),, 7.59 \quad(1 \mathrm{H}, \mathrm{t}$, $\mathrm{CH}=\mathrm{N}), 6.37(1 \mathrm{H}, \mathrm{s}, \mathrm{C}=\mathrm{CH}), 3.52(3 \mathrm{H}, \mathrm{s},-\mathrm{OMe}), 2.07\left(2 \mathrm{H}, \mathrm{q},-\mathrm{CH}_{2,}\right), 1.31\left(2 \mathrm{H}, \mathrm{sext},-\mathrm{CH}_{2}\right)$, $0.69\left(3 \mathrm{H}, \mathrm{t},-\mathrm{CH}_{3}\right),{ }^{13} \mathrm{C}$ NMR (DMSO) $\delta: 165.80,163.83,143.01,113.98,52.37$ (-OMe), 34.03 ($\left.\mathrm{CH}_{2-}\right), 18.92\left(-\mathrm{CH}_{2-}\right) 13.57\left(-\mathrm{CH}_{3}\right), \mathrm{MS}(\mathrm{m} / \mathrm{z}, \%): 255$ (parent peak), 85 (base peak, 100\%), 227 , 187, 59, 43. Anal. Calcd for $\mathrm{C}_{10} \mathrm{H}_{13} \mathrm{~N}_{3} \mathrm{O}_{3} \mathrm{~S}$ : C, 47.06; H, 5.10; N, 16.47\%. Found; C, 46.88; H, $4.90 ; \mathrm{N}, 16.23 \%$.

\{4-Oxo-2-[(1-P-tolyl-ethylidene)-hydrazono]-thiazolidin-5-ylidene\}-acetic acid ethyl ester (7c).Yellow solid ,1.15 g, yield 87\%, m.p $>220{ }^{0} \mathrm{C}$, IR (KBr) $\left(\hat{v}_{\max }, \mathrm{cm}^{-1}\right): 2956-3081(\mathrm{CH})$, $3180(\mathrm{~N}-\mathrm{H}), 1741,1716(\mathrm{C}=\mathrm{O}), 1641,1617(\mathrm{C}=\mathrm{N}),{ }^{1} \mathrm{H}$ NMR (DMSO) $\delta: 11.75(1 \mathrm{H}, \mathrm{s}, \mathrm{N}-\mathrm{H})$, 7.74-7.23 (4H, 2d, arom), $6.58(1 \mathrm{H}, \mathrm{s}, \mathrm{CH}=\mathrm{C}), 4.21\left(2 \mathrm{H},-\mathrm{CH}_{2-}\right), 2.47\left(3 \mathrm{H},-\mathrm{CH}_{3}\right) 2.33\left(3 \mathrm{H},-\mathrm{CH}_{3}\right)$, $1.25\left(3 \mathrm{H},-\mathrm{CH}_{3}\right),{ }^{13} \mathrm{C}$ NMR (DMSO) $\delta: 165.31,165.08(2 \mathrm{C}=\mathrm{O}), 162.55,158.28(2 \mathrm{C}=\mathrm{N}) 142.63$ $(\mathrm{C}=\mathrm{CH}), 139.85,134.44,128.78,126.29(6 \mathrm{C}$, arom $), 114.25(\mathrm{C}=\mathrm{CH}), 60.95\left(-\mathrm{CH}_{2}\right), 20.62(-$ $\mathrm{CH}_{3}$-para), 14.47(- $\left.\mathrm{CH}_{3}\right) 13.71\left(-\mathrm{CH}_{3}\right), \mathrm{MS}(\mathrm{m} / \mathrm{z}, \%): 331$ (parent peak), 118 (base peak, 100\%), 316, 91, 65. Anal. Calcd for $\mathrm{C}_{16} \mathrm{H}_{17} \mathrm{~N}_{3} \mathrm{O}_{3} \mathrm{~S}$ : C, 58.00; H, 5.14; N, 12.69\%. Found; C, 58.21; H, $5.19 ; \mathrm{N}, 12.47 \%$.

\{4-Oxo-2-[(1-P-tolyl-ethylidene)-hydrazono]-thiazolidin-5-ylidene\}-acetic acid methyl ester (8c). Yellow solid ,1.14 g, yield 90\%, m.p >220 ${ }^{\circ} \mathrm{C}$ dec., $(\mathrm{KBr})\left(\hat{v}_{\max }, \mathrm{cm}^{-1}\right): 3180(\mathrm{~N}-\mathrm{H}), 3081(\mathrm{C}-$ $\mathrm{H}$, arom) 1741, $1716(\mathrm{C}=\mathrm{O}), 1641,1617(\mathrm{C}=\mathrm{N}),{ }^{1} \mathrm{H}$ NMR (DMSO) $\delta: 12.76(1 \mathrm{H}, \mathrm{s}, \mathrm{N}-\mathrm{H}), 7.76-$ $7.22\left(4 \mathrm{H}, 2 \mathrm{~d}\right.$, arom), $6.61(1 \mathrm{H}, \mathrm{s}, \mathrm{CH}=\mathrm{C}), 3.75(3 \mathrm{H}, \mathrm{s},-\mathrm{OMe}), 2.39\left(3 \mathrm{H}, \mathrm{s},-\mathrm{CH}_{3}\right), 2.33(3 \mathrm{H}, \mathrm{s},-$ $\left.\mathrm{CH}_{3}\right),{ }^{13} \mathrm{C}$ NMR (DMSO) $\delta: 165.88,165.53,(2 \mathrm{C}=\mathrm{O}), 162.08,143.14(2 \mathrm{C}=\mathrm{N}), 140.14,140.00$, 134.48, 129.06, 126.50, $114.00(\mathrm{C}=\mathrm{CH}), 52.17,20.39,14.74 . \mathrm{MS}(\mathrm{m} / \mathrm{z}, \%): 317$ (parent peak, 100\%), 118 (base peak), 302, 91, 65. Anal. Calcd for $\mathrm{C}_{15} \mathrm{H}_{15} \mathrm{~N}_{3} \mathrm{O}_{3} \mathrm{~S}: \mathrm{C}, 56.78 ; \mathrm{H}, 4.73 ; \mathrm{N}$, 13.25\%. Found; C, 56.57; H, 4.67; N, 13.01\%.

\{2-[(4-Methoxy-benzylidene)-hydrazono]-4- oxo-thiazolidin-5-ylidene\}-acetic acid ethyl ester (9c).Yellow solid,1.13 g, yield 85\%, m.p >220 ${ }^{\circ} \mathrm{C}$ dec., $(\mathrm{KBr})\left(\hat{v}_{\max }, \mathrm{cm}^{-1}\right)$ : 2982-3000 , 1741, $1716(\mathrm{C}=\mathrm{O}), 1666,1617(\mathrm{C}=\mathrm{N}),{ }^{1} \mathrm{H}$ NMR (DMSO) $\delta: 8.37(1 \mathrm{H}, \mathrm{s}, \mathrm{CH}=\mathrm{N}), 7.74-6.93(4 \mathrm{H}$, 2d, arom), 6.78, $(1 \mathrm{H}, \mathrm{s}, \mathrm{CH}=\mathrm{C}), 4.31\left(2 \mathrm{H}, \mathrm{q},-\mathrm{CH}_{2}-\right), 3.85\left(3 \mathrm{H}, \mathrm{s},-\mathrm{OCH}_{3}\right), 1.35\left(3 \mathrm{H}, \mathrm{t},-\mathrm{CH}_{3}\right){ }^{13} \mathrm{C}$ NMR (DMSO) $\delta: 170.94,166.02,(2 \mathrm{C}=\mathrm{O}), 166 . \mathrm{O} 4,162.02(2 \mathrm{C}=\mathrm{N}), 159.48,158.13(2 \mathrm{C},-\mathrm{S}-$ $\mathrm{C}=\mathrm{CH}), 60.24,\left(-\mathrm{CH}_{2}-\right), 55.39(-\mathrm{OMe}), 14.17\left(1 \mathrm{C},-\mathrm{CH}_{3}\right) . \mathrm{MS}(\mathrm{m} / \mathrm{z}, \%: 333$ (parent peak), 134 (base peak, 100\%),120, 91, 77, 57.Anal. Calcd for $\mathrm{C}_{15} \mathrm{H}_{15} \mathrm{~N}_{3} \mathrm{O}_{3} \mathrm{~S}: \mathrm{C}, 54.05 ; \mathrm{H}, 4.20 ; \mathrm{N}, 12.61 \%$. Found; C, 53.94; H, 4.17; N,12.48\%.

\section{Acknowledgements}

The authors express their gratitude to the Shahid Bahonar University of Kerman Faculty Research Funds for support of this investigation. 


\section{References and Footnotes}

1. Elgemeie, G. H.; Sayed, S. H. Synthesis 2001, 12, 1747.

2. Berseneva, V. S.; Tkachev, A.V.; Morzherin, Yu.Yu.; Dehaen, W.; Luyten, I.; Toppet, S.; Bakulev, V. J. Chem. Soc., Perkin Trans I. 1998, 15, 2133.

3. Rudnichenko, A.V.; Timoshenko, V. M.; Shermolovich, Yu. G. Journal of Fluorine Chemistry 2004, 125, 439.

4. Rudnichenko, A. V.; Timoshenko, V. M.; Chernega, A. N.; Nesterenko, A. M.; Shermolovich, Yu. G. ibid. 2004, 125, 1351.

5. Acheson, R. M.; Wallis, J. D. J. Chem. Soc., Perkin Trans I. 1981, 2, 415.

6. Loupy, A. Microwaves in organic synthesis, Wiley-VCH; 2006.

7. Sheibani, H.; Mosslemin, M. H.; Behzadi, S.; Islami, M. R.; Foroughi, H.; Saidi, K. ARKIVOC, 2005, 15, 88.

8. Maslen H. L.; Hughes, D.; Hursthouse, M.; De Clercq, E.; Balzarini, J.; Simons, C. J. Med. Chem. 2004, 47, 5482. 